\title{
High resolution temperature independent refractive index measurement using differential white light interferometry
}

\author{
Carlos Gouveia a,b,*, Mohammad Zibaii ${ }^{c}$, Hamid Latifi $^{c}$, Manuel J.B. Marques ${ }^{\mathrm{d}}$, \\ J.M. Baptista ${ }^{\mathrm{a}, \mathrm{b}, 1}$, Pedro A.S. Jorge ${ }^{\mathrm{a}, * *}$ \\ a INESC Porto, Rua do Campo Alegre, 687, 4169-007 Porto, Portugal \\ b Centro de Competências de Ciências Exactas e de Engenharia, Universidade da Madeira, Campus da Penteada, 9000-390 Funchal, Portugal \\ ${ }^{\mathrm{c}}$ Laser and Plasma Research Institute, Shahid Beheshti University, Evin, Tehran, Iran \\ ${ }^{\mathrm{d}}$ FCUP, Rua do Campo Alegre, 687, 4169-007 Porto, Portugal
}

\section{A R T I C L E I N F O}

\section{Article history:}

Received 21 February 2013

Received in revised form 6 August 2013

Accepted 7 August 2013

Available online $\mathrm{xxx}$

\section{Keywords:}

Fiber optic sensors

White light interferometry

Differential measurement

Virtual instrumentation

Tapers

Refractive index measurement

\begin{abstract}
A B S T R A C T
In this work a fiber optic interferometric system for differential refractive index measurement is described. The system is based on a white light Mach-Zehnder configuration, with serrodyne phase modulation, used to interrogate two similar non-adiabatic tapered optical fiber sensors in a differential scheme. In this situation the system is able to measure the refractive index independent of temperature. Signal processing with low cost digital instrumentation developed in Labview environment allows a detectable change in refractive index of $\Delta n \approx 1.46 \times 10^{-6}$, which is, from the best of our knowledge the highest resolution achieved using a bare fiber taper device for a range of refractive index close to the water index. The results demonstrate the potential of the proposed scheme to operate as a self-referenced chemical and biological sensing platform.
\end{abstract}

(C) 2013 Elsevier B.V. All rights reserved.

\section{Introduction}

The measurement of chemical and biological parameters in diversified environments is currently recognized as an important issue for a diversity of complex systems ranging from industrial processes, medicine and environmental applications. In this context, label free optical sensing based on the measurement of refractive index (RI) represents an interesting solution. Such approaches do not interfere with the analyte properties and require, instead, the design of sensitive layers that experience a refractive index change in its presence. This can be achieved by using biomolecules with a natural affinity to the target, or chemical species having analyte specific ligands. The combination of such membranes with refractive index sensors can therefore provide attractive solutions for biochemical sensing [1].

\footnotetext{
* Corresponding author at: INESC Porto, Rua do Campo Alegre, 687, 4169-007 Porto, Portugal. Tel.: +351 220402 301; fax: +351 220402437.

** Corresponding author. Tel.: +351 220402 301; fax: +351220 402437 .

E-mail addresses: cgouveia@fc.up.pt, cgouveia@inescporto.pt, icarlos07@gmail.com (C. Gouveia), pedro.jorge@fc.up.pt (P.A.S. Jorge).

1 Tel.: +351220 402 301; fax: +351220402437.
}

Fiber optic sensors, in particular, are an interesting solution due to their high sensitivity, small size, and capability for in situ, real-time, remote, and distributed sensing. Several schemes for refractive index sensing using optical fibers have already been proposed. Surface plasmon resonance (SPR) is one of the most common [2-4], other solutions include the use of non-adiabatic fiber tapers [5], core exposed fiber Bragg grating (FBG) [6], long period gratings (LPG) [7] or LPG based interferometers [8]. In these reported works most devices present a high sensitivity to the surrounding refractive index. However, all of these configurations are also sensitive to temperature. Furthermore, some of these sensors are more sensitive to temperature than to refractive index.

Temperature cross-sensitivity is a characteristic which affect in greater or lesser degree all the optical fiber sensors, this arises from intrinsic sensitivity of the silica matrix to temperature, through the thermo-optic and photo-elastic coefficients, which induce changes in the effective refractive index and the effective length of the fiber, respectively, thus, varying the optical signal as a function of the thermal conditions. These changes may be higher or lower, depending on the type of optical device used and on the evaluated optical parameter. Typically, the thermo-optical coefficient of silica is $8.0 \times 10^{-6} \mathrm{~K}^{-1}$ and its thermal expansion coefficient has the value of $0.55 \times 10^{-6} \mathrm{~K}^{-1}$, these figures may change according with the composition of the material [9]. Therefore, the influence 
of temperature must be accounted for when high resolution RI measurements are carried out for high sensitivity detection of biological or chemical parameters. Though, in most cases authors deal with temperature dependence working with temperature stabilized systems. Nevertheless, even when temperature is stabilized to $\pm 0.1^{\circ} \mathrm{C}$, such fluctuations can still be significant in face of the need to measure variation of refractive index in the order of $10^{-5}$ or less.

Therefore, the desirable situation is to have a system that can either compensate or simultaneously measure temperature. During the last few years several fiber optic refractometric configurations for simultaneous measurement of temperature and RI were presented. Most of the solutions rely on the use of a pair of gratings: cascaded LPGs with different RI and temperature sensitivities and thereby capable of measuring the surrounding refractive index (SRI) without ambiguity [10] or two FBGs where one of them was side polished to become RI sensitive and the other serves as temperature reference [11]. A Hybrid configuration using a pair of FBGs and one LPG was also presented [12]. Recently, a different approach was demonstrated employing a fiber loop mirror (FLM) with two section of polarizing maintaining fiber, one etched to be RI sensitive and the other one used as a thermal reference [13]; using a similar strategy a combination of a multimode interferometer (MMI) refractometer device into a FLM was also used for this purpose [14].

In the vast majority of these works the sensors were interrogated in the spectral domain using devices such as optical spectrum analyzers. However, the use of interferometric methods can further increase the measurement resolution. White Light Interferometry (WLI) is one of the most attractive interferometric interrogation methods used and it has been applied for remote measurement of diverse parameters such as strain, temperature, pressure and refractive index [15-18]. This technique uses low coherence optical sources providing absolute measurement at high resolution and the ability of multiplexing sensors onto a single optical fiber using coherence multiplexing [19-21]. An issue associated with this readout technique, however, is the sensitivity of the readout interferometer to external and internal perturbations such as vibrations, temperature, among others. These perturbations can induce significant phase drifts limiting the resolution of the measurements. It is possible to decrease those phase deviations by properly isolating the readout interferometer. However, due to its high sensitivity, this solution is not entirely effective. In this context, using a Mach-Zhender configuration it is possible to set up a differential measurement scheme through the comparison of the phase of two output signals of the interferometer. In such case the environmentally induced phase drifts will be canceled out (common phase noise rejection) allowing a very stable and accurate phase measurement. Such system is then a powerful tool to track the wavelength changes of external interferometric sensors. In particular, it can be used to interrogate two similar sensor interferometers simultaneously, one as the measurement sensor and a second one as a reference sensor. Using appropriate setting of the path imbalances of each of the interferometers the phase difference between the two signals depends only on the modulation imposed by the parameter of interest, thereby reducing also the effects of cross-sensitivity, indissociable of these systems. Differential WLI system have been employed to measure temperature [22,23], liquid refractive index $[24,25]$, air refractive index [26] and for fuel conformity analysis [27].

In this work an interferometric readout system, controlled with virtual instrumentation, is described where a Mach-Zehnder white light system is used to interrogate two similar non-adiabatic fiber tapers in a differential arrangement. In this configuration it is possible to measure the refractive index with high resolution and independent of temperature. The results demonstrate the potential of the proposed scheme to operate as a high-resolution label-free bio-chemical sensing platform avoiding the need for temperature control.

\section{Principle and experimental procedure}

Fig. 1 shows the setup of the readout interferometer which is in a standard fiber optic Mach-Zehnder configuration where the sensing and reference interferometers are connected to each of the output arms. For proper operation the path imbalance of the reading interferometer should match that of the sensor interferometers so that the overall path is nearly zero. For this purpose, in one of the arms of the Mach-Zehnder, an open air path is implemented through a circulator using a graded-index (GRIN) lens and a mirror mounted in a translation stage. With this arrangement the reading interferometer path imbalance can be tuned to match the optical path difference of the sensing interferometers. On the other arm, an electro-optical phase modulator (APE from JSDU) was inserted for carrier generation, where a sawtooth modulation with its amplitude adjusted to obtain a $2 \pi$ phase excursion was used yielding a sinusoidal carrier generation at the interferometer output. The coupling ratio of the first coupler was chosen in order to maximize the fringe visibility of the interferometer. Due to high insertion loses of the phase modulator $(4 \mathrm{~dB})$ compared with the open air path ( $2 \mathrm{~dB})$, a coupling ratio of 70:30 is used. In order to keep the readout device isolated from external environmental perturbation such as temperature and vibration a proper packaging was carefully done using insulating materials.

The two output ports of the readout interferometer are used to interrogate two similar interferometric sensors, which are subsequently connected to two photodetectors. In this arrangement, the readout interferometer acts as a modulator for the two sensing interferometers conditioning the signal received by the photodetectors. When the optical path difference (OPD) of the sensors is matched to the readout interferometer within the coherence length of the source, it is possible to get the phase information of the sensing probes at the frequency of the carrier. Using one of the interferometers as reference and the other as sensor, and since the Mach-Zehnder outputs are in phase opposition, most environmentally induce phase drifts can be canceled out, and the phase difference between both signals depends only on the modulation inferred by the parameter of interest allowing a very stable and accurate phase measurement retrieved using phase comparison software.

In this experiment the interferometers used as sensing devices were non-adiabatic tapered optical fibers (NATOF). The non adiabatic fiber tapers can be fabricated in such a way that coupling occurs primarily between the fundamental mode of the un-pulled fiber and higher order modes of the taper waveguide, where due to the large difference of the refractive indexes of air and fiber cladding, the taper normally supports more than one mode. The light propagates at the air-cladding interface of the tapers waist region in which case the single-mode fiber (SMF) is converted into a multimode waveguide. The result of back and forth coupling between the single mode of the fiber and the two (or more) modes of the taper is an oscillatory spectral response. The efficiency of this last coupling is dependent on the relative phase of the participating modes. Therefore, a NATOF behaves as a Mach-Zehnder modal interferometer [28]. Fig. 2 shows a conceptual scheme of the sensing probe (A) and its behavior as a two waves interferometer (B). When there are only two modes, the relative phase at the output is given by $\Delta \varphi=\left(n_{\text {eff,co }}-n_{\text {eff,cl }} \mathrm{m}\right) L$, where $n_{\text {eff,co }}$ and $n_{\text {eff,cl }} \mathrm{m}$ are the effective indices of the core and $m$ th cladding mode respectively and $L$ is interaction length along the taper. Therefore, the spectral response of the taper will shift correspondingly by changing the above terms. For instance, if the RI of the surrounding environment 


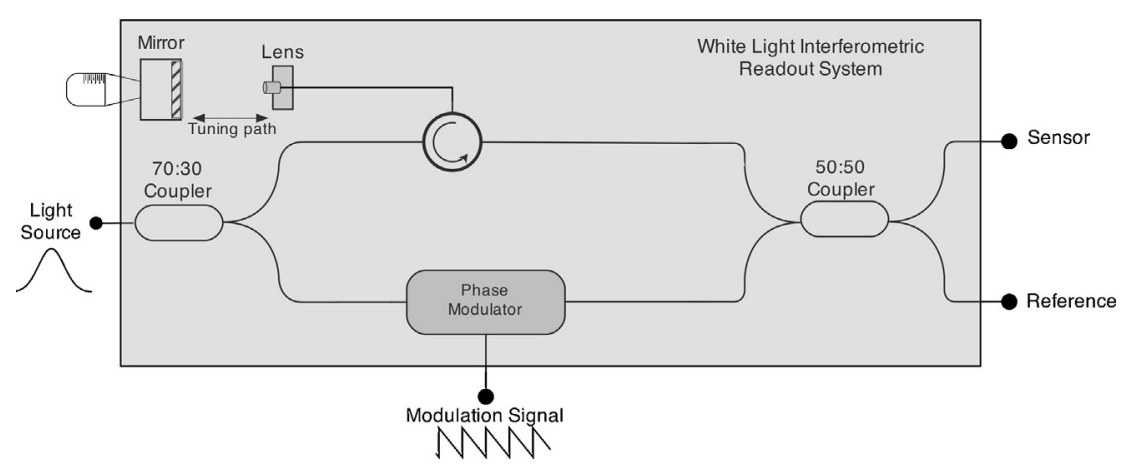

Fig. 1. White light interferometry based readout device.

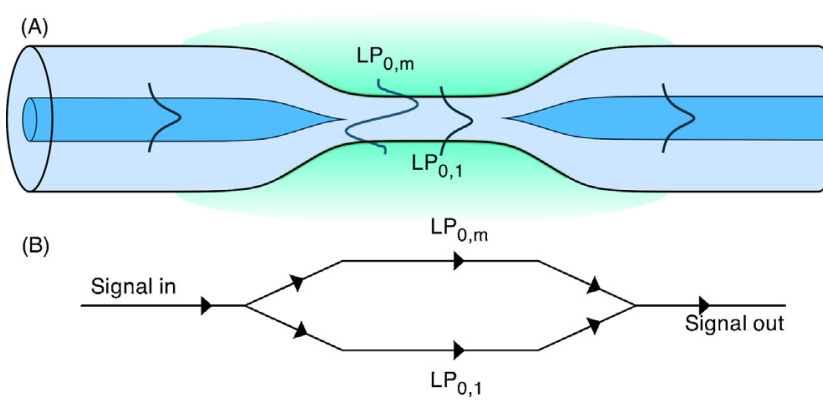

Fig. 2. (a) Schematic representation of non-adiabatic taper. (b) Conceptual representation as a modal Mach-Zehnder interferometer.

of the taper changes, the difference in effective index of the cladding mode and the relative phase would be modified leading to a shift of the spectral response. NAFOF based fiber refractometers have been used recently as refractometers in the biochemical field due to its high sensitivity and compact size $[5,29,30]$.

Fig. 3 shows the experimental setup for differential measurement of refractive index. The readout interferometer was illuminated by a superluminescent diode (SLD) source $\left(\Delta \lambda_{\mathrm{FWHM}} \approx 50 \mathrm{~nm}\right.$ and $\left.L_{\text {coherence }} \approx 15 \mu \mathrm{m}\right)$. A sawtooth modulation signal $(1 \mathrm{kHz})$ with amplitude $7.2 \mathrm{~V}$ generated by a signal acquisition board (DAQ NI 6259 USB) was applied. Two similar NATOFs connected to its output ports were used. The sensing devices were fabricated with heat pulling method using a $\mathrm{CO}_{2}$ laser. The experiment was performed using NATOFs with a taper length around $20 \mathrm{~mm}$, with a transition region of about $2 \mathrm{~mm}$ and a uniform waist length approximately of $16 \mathrm{~mm}$ with an average taper waist diameter in the range of 6-8 $\mu \mathrm{m}$. The optical power loss of the tapers were $9 \%$. Before characterization, the spectra of both tapers were obtained by using a simple setup, which consists in a SLD light source (the same used in the experiment) to illuminate the sensors, meanwhile the sensing devices output were plugged to an Advantest Q8384 optical spectrum analyzer (OSA) with $10 \mathrm{pm}$ maximum resolution, where the spectra were recorded. Fig. 4 shows the spectra of the two sensing probes. A very smooth fringe pattern is observed in both cases, modulated by the envelope of the optical source. Similar fringe periods and fringe visibility are observed in both cases, indicating that the sensors are almost identical interferometers making this set suitable to use as a sensor/reference pair.

The sensing elements were placed into a test chamber with both fiber ends properly fixed to avoid strain/curvature crosssensitivity. The reference element was inside of a $260 \mu \mathrm{m}$ diameter metallic capillary tube, thereby sensitive to temperature changes in the solution but insensitive to the refractive index variations in the solution. The transmitted signal was guided toward two photodetectors plugged into an acquisition board. Then, at the signal processing stage, the output of the detectors is filtered through a band-pass filter centered at modulation frequency, thereby eliminating the remaining higher harmonic components (created by discontinuities induced by sawtooth modulation signal). The filter introduces a fixed bias on the phase of both signals. Therefore, the differential phase measurement is not affected by the constant phase deviation introduced by the filtering. In this situation, after filtering, the changes in the surrounding refractive index

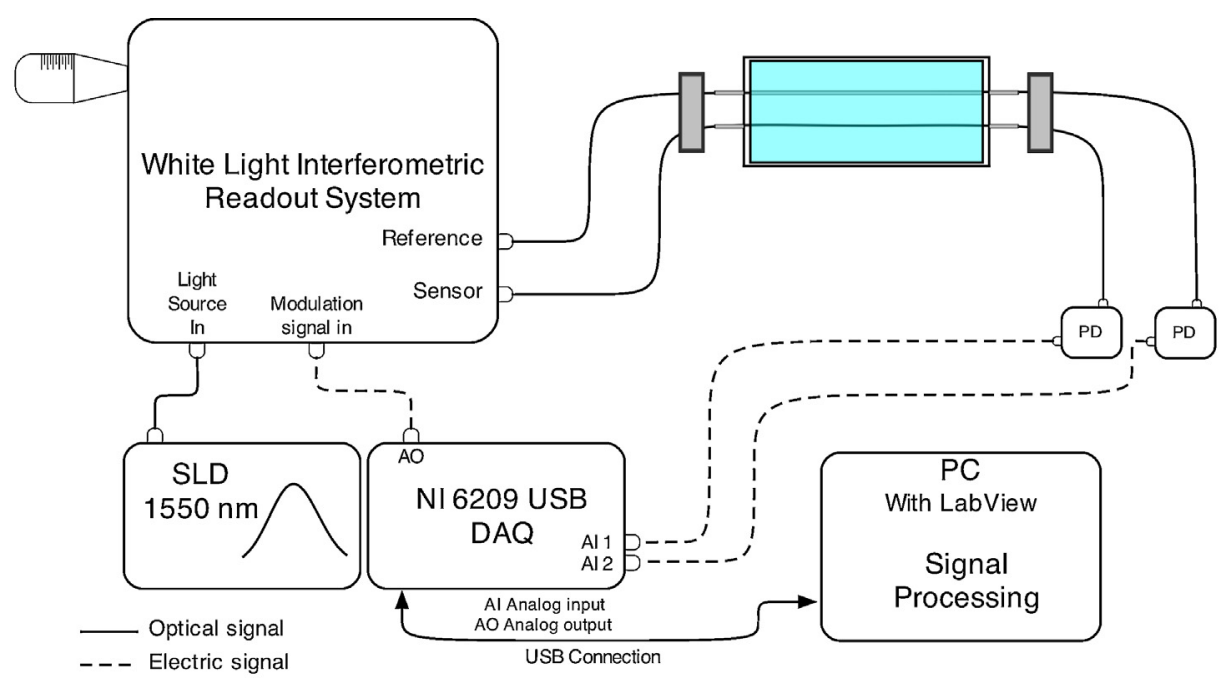

Fig. 3. Setup for differential measurement of refractive index using a readout interferometer and two similar taper devices. 


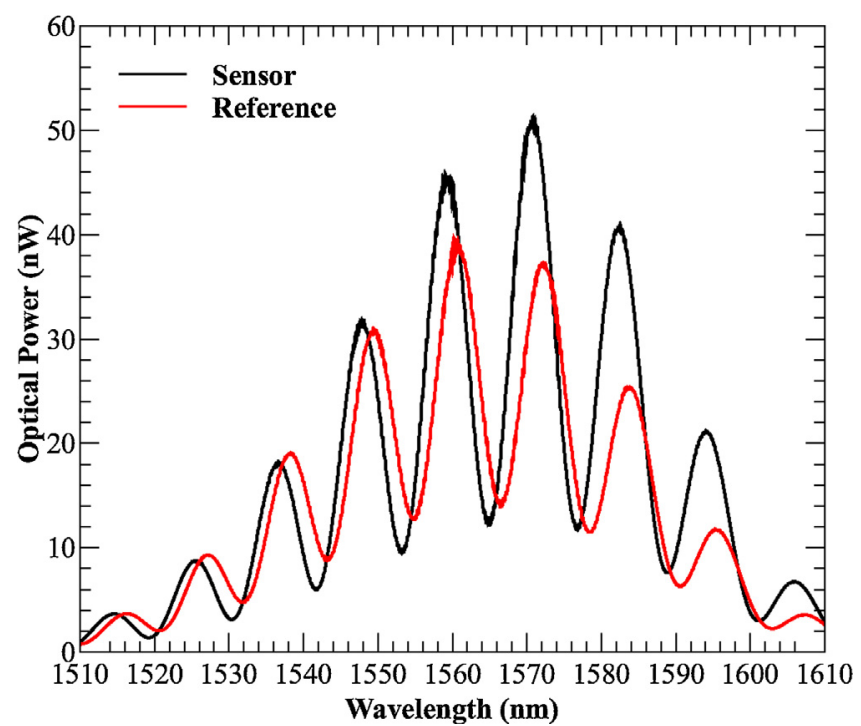

Fig. 4. Transmitted spectra of the two interferometric devices used in the experiment.

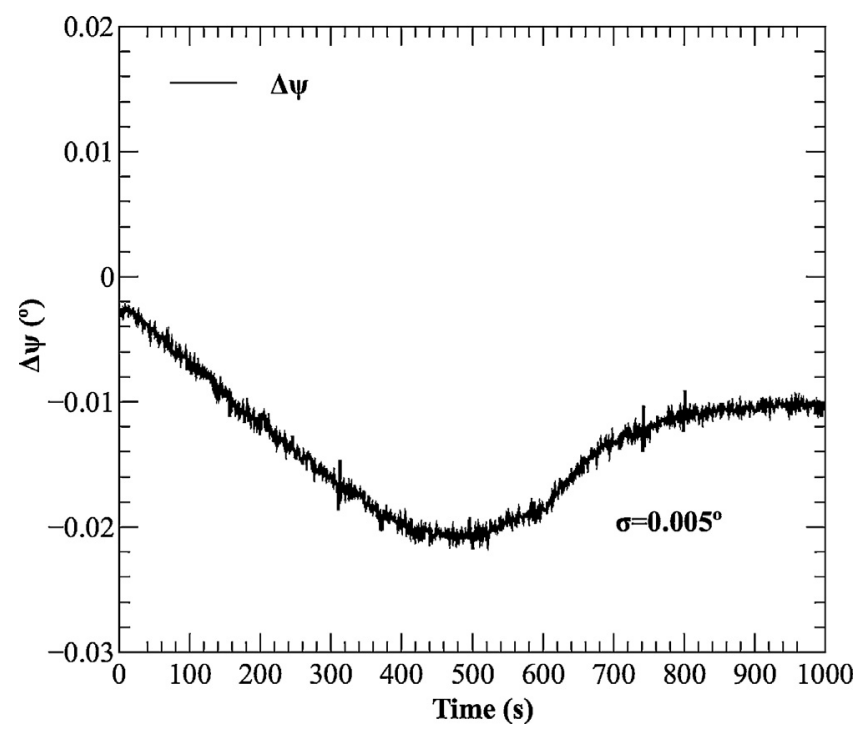

Fig. 5. Stability of the differential phase between the two output ports of the readout device.

output arise as a phase shift on the sinusoidal carrier signal, which is retrieved using the relative phase comparison software. The differential phase signal is therefore sensitive only to the refractive index of the solution.

\section{Results and discussion}

Prior to the refractive index characterization, the intrinsic stability of the readout interferometer was investigated by measuring the differential phase $(\Delta \psi)$ between the two output ports, with no sensor plugged in. The two arms relative phase was monitored for a period of time of approximately thousand seconds. Fig. 5 shows the recorded phase response during the tested time interval. From the statistics of the recorded values the standard deviation was calculated. This data shows the residual random drift of the interrogation system, which is very small $(\sigma \sim 0.0045)$ proving the effectiveness of common noise rejection of the differential scheme. This result shows that the system is able to resolve a minimum phase change of $\pm 0.009^{\circ}$ (considering the resolution is given by $2 \sigma$ ).

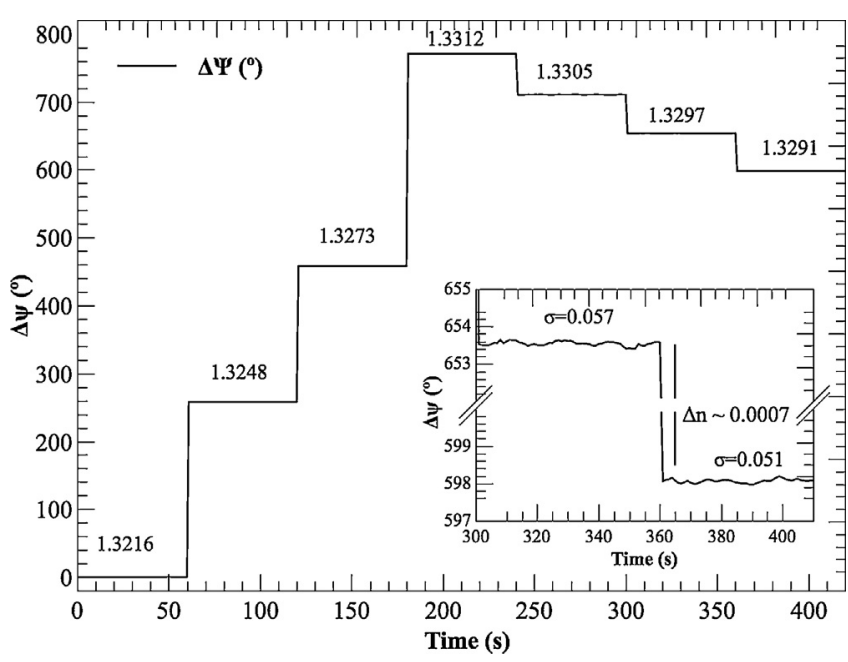

Fig. 6. Time response of the sensing system to external refractive index changes The inset shows with more detail the phase difference fluctuation for a change in SRI from 1.3305 and 1.3312 .

Following this initial test, the NATOF sensors were plugged into the Mach-Zhender outputs and tested for refractive index measurements. The response of the sensor to the surrounding RI was studied by exposing the sensing head to different solutions of distilled water mixed with different concentrations of ethylene glycol to provide the RI standards in a range between 1.3216 and 1.3312 . The refractive index was changed by successively adding different ratios of ethylene glycol/deionized water. In order to homogenize the mixture, the solution was stirred through a magnetic stirrer. After mixing, the stirring was stopped and the data was recorded, the process was repeated continuously for each of step .The liquid samples were characterized by an Abbe refractometer using the sodium $\mathrm{D}$ line $(589 \mathrm{~nm})$. The necessary adjustments, considering the sensing head operation at $1550 \mathrm{~nm}$, were made using the Cauchy equation with the respective coefficients [31,32]. The real time response of the differential sensing platform to surrounding refractive index (SRI) step changes is shown in Fig. 6. When acquiring this data, the recording was paused during the changing of the solutions, being resumed after $100 \mathrm{~s}$. This way all the mechanical perturbation introduced by the changing of the solutions was avoided. In a real application the sensors are bound to be inserted in flow system were this is not a problem. The results show, as expected, an increment in the phase difference with the increase of the SRI. From this data a calibration curve can be obtained that is showed in Fig. 7. A sensitivity of $80,084 \pm 190 \mathrm{RIU}^{-1}$ can be estimated from the slope of the acquired this data. The inset in Fig. 6 shows with more detail the phase difference fluctuation for a change in SRI from 1.3312 and 1.3305. From the statistics of the measured values the standard deviation was calculated for each step. It was then considered a minimum detectable signal of two times the average calculated standard deviation $(\sigma)$ divided by the sensitivity in the measurement interval. From this procedure an average refractive index resolution of $\pm 1.46 \times 10^{-6}$ RIU was calculated. This value is, from the best of our knowledge the highest resolution achieved using a bare fiber taper device for a range of refractive index close to the water index. In addition, in the best measurement obtained a resolution of $\pm 6 \times 10^{-7}$ RIU was achieved indicating the potential of this configuration to attain the highest resolution. In a practical application more stringent statistics can be applied. In such case, for instance a $3 \sigma$ standard would yield an average resolution of $\pm 2.16 \times 10^{-6} \mathrm{RIU}$. Although a resolution of $\pm 7 \times 10^{-7}$ RIU was reported by Monzon-Hernandez et al. [4] in this case the authors used an additional metal coating to obtain an SPR sensor over a fiber taper. In addition the measurements were 


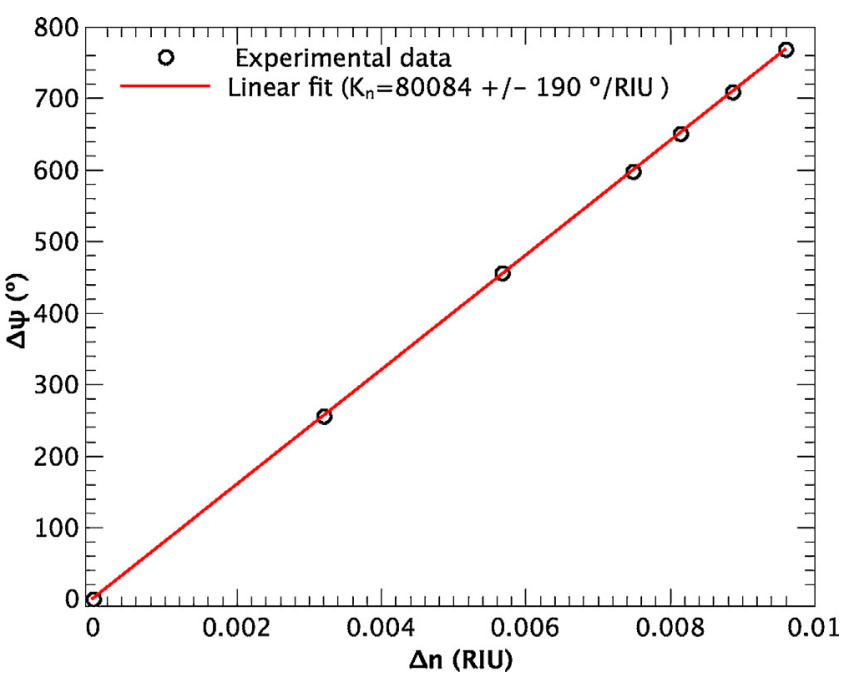

Fig. 7. Calibration curve of the system for refractive index variations.

performed for a range of RI close to the cladding index where sensitivity is higher. Also, the resolution was estimated considering the resolution of the OSA and the accurate measurement of the SPR peak, that in our modest opinion, neglects several factors namely; the resonance bandwidth and signal fluctuations inherent to the measure itself due to temperature variation, low optical power, among others all concurring to decrease the final system resolution.

The small variations observed in the resolution calculated in different intervals of refractive index are probably due to actual refractive index fluctuations arising from heterogeneity in the dissolution of ethylene glycol in water. This is partially confirmed by the fact that highest resolution values were obtained for the solutions were the concentration of ethylene glycol was smaller. Also further improvements are possible by enhancing the interrogation interferometer stability. Having a birrefringent element inside the interferometer (the phase modulator) introduces some polarization dependent noise. Therefore, active polarization control, or alternative way of modulate the interferometer phase, can potentially improve the results obtained.

Regarding the temperature insensitivity is important to clarify that, in the present arrangement the differential measurement only cancels out the effect of temperature on the fiber sensor itself. This sensor arrangement is still sensitive to the changes induce in the refractive index of the solution by the temperature, i.e. variations of refractive index derived from the thermo-optic coefficient of the solution will be measured while the effects of the silica thermo-optic coefficient in the fiber sensor are canceled out. This happens because the reference sensor is inside the capillary with no contact with the solution. This is an important feature as many chemical reactions show exothermal behavior which could be, this way, accurately tracked by measuring the corresponding refractive index changes.

It is important to emphasize that in the present configuration and for the achieved resolution, there is a limitation for the temperature independent measurement, which arises from the fact that the reference sensor was in air. Assuming that the intrinsic sensitivity characteristics of both taper are very close each other still the fact that they are in different media limits the extent of compensation. The reference element is sensitive to variations in the thermo-optic coefficient of the air, which is around $10^{-6}$ [33]. On the other hand it is well known that the sensitivity to the SRI of the evanescent field based sensors, shows a nonlinear monotonic behavior with increasing sensitivity as the SRI approaches the cladding RI. It means that the sensitivity of the taper for a refractive index around 1 will be lower than for a RI close to 1.32 Some simple calculations performed to evaluate this difference revealed that, the sensitivity of the device for a SRI near to 1 is 6.6 times lower, than for SRI close to 1.32. Thus, it is possible to assert that, in the current configuration, it is possible to have an independent temperature measurement within the estimated resolution, for the variation lower than $10^{\circ} \mathrm{C}$. For higher temperature changes, and since all the coefficients involved are known it is possible to extend the compensation range by appropriate calibration and software correction.

In the cases where the thermo-optic coefficient needs to be compensated, the reference sensor has to be in contact with the solution. In the present configuration, this can be easily done by filling the capillary tube with deionized water. In such a case the sensing platform will be able to measure the refractive index variations in the sample. Such platform will enable to implement self-referenced biosensors where the measurement can be made independent of temperature, bulk refractive index changes and non-specific binding events at the surface.

Considering all the described features, the proposed sensing system demonstrates to be suitable for very high sensitivity refractometric measurement without temperature ambiguity, for both chemical and biological sensing applications. For instance, direct measurements of refractive index were shown to provide valuable information on drug/DNA interaction, or cell growth [5,29]. On the other hand, a variety of silica surface functionalization is described in the literature that will render a refractometer a powerful biosensor [1]. In such case, both sensing devices may be in contact with the solution, the reference can be functionalized with a passive layer enabling, therefore, to compensate for temperature, bulk refractive index and even non-specific binding events enabling a truly self-referenced biosensor.

\section{Conclusions}

In this work a differential interferometric scheme for interrogation of fiber optic interferometric refractometers was implemented. Differential measurements of refractive index were carried out using two similar NATOFs. One of the tapers was used as sensor and was exposed to variations of SRI and temperature and a second taper was used as a reference and exposed only to the thermal variations in the sample chamber. In this arrangement the system is able to measure the refractive index independent of temperature. The sensing system was characterized in the range between 1.3216 and 1.3312 RIU. A sensitivity of $84,084 \pm 190$ RIU $^{-1}$ was estimated and an average resolution of $\pm 1.46 \times 10^{-6}$ RIU was achieved $\left( \pm 6 \times 10^{-7} \mathrm{RIU}\right.$ in the best case). It was demonstrated, by the sensitivity and resolutions obtained that the proposed configuration is a sensing system with very high performance for label-free sensing applications. In biosensing applications, the reference can be functionalized with a passive layer enabling, therefore, to compensate for temperature, bulk refractive index and even non-specific binding events enabling a truly self-referenced biosensor.

\section{Acknowledgements}

This work was supported by project AQUAMONITOR $\mathrm{n}^{\circ}$ FCOMP01-0124-FEDER-013911 (Ref. PTDC/AAC-AMB/112424/2009). Carlos Gouveia would like to acknowledge the financial support of FCT (SFRH/BD/63758/2009).

\section{References}

[1] X. Fan, I.M. White, S.I. Shopova, H. Zhu, J.D. Suter, Y. Sun, Sensitive optical biosensors for unlabeled targets: a review, Anal. Chim. Acta 620 (2008) 8-26. 
[2] R. Slavík, J. Homola, J. Ctyroky, E. Brynda, Novel spectral fiber optic sensor based on surface plasmon resonance, Sens. Actuators B (2001) $106-111$.

[3] N. Díaz-Herrera, A. Gonzalez-Cano, D. Viegas, J.L. Santos, M.C. Navarrete, Refractive index sensing of aqueous media based on plasmonic resonance in tapered optical fibres operating in the $1.5 \mu \mathrm{m}$ region, Sens. Actuators B 146 (2010) 195-198.

[4] D. Monzon-Hernandez, J. Villatoro, High-resolution refractive index sensing by means of a multiple-peak surface plasmon resonance optical fiber sensor, Sens. Actuators B 115 (2006) 227-231.

[5] M.I. Zibaii, A. Kazemi, H. Latifi, M.K. Azar, S.M. Hosseini, M.H. Ghezelaiagh, Measuring bacterial growth by refractive index tapered fiber optic biosensor, J. Photochem. Photobiol. B 101 (2010) 313-320.

[6] A. Asseh, S. Sandgren, H. Ahlfeldt, B. Sahlgren, R. Stubbe, G. Edwall, Fiber optical Bragg grating refractometer, Fiber Integr. Opt. 17 (1998) 51-62.

[7] H.J. Patrick, A.D. Kersey, F. Bucholtz, Analysis of the response of long period fiber gratings to external index of refraction, J. Lightwave Technol. 16 (1998) $1606-1612$.

[8] T. Allsop, R. Reeves, D.J. Webb, I. Bennion, R. Neal, A high sensitivity refractometer based upon a long period grating Mach-Zehnder interferometer, Rev. Sci. Instrum. 73 (2002) 1702-1705.

[9] A.D. Kersey, M.A. Davis, H.J. Patrick, M. LeBlanc, K.P. Koo, C.G. Askins, et al., Fiber grating sensors, J. Lightwave Technol. 15 (1997) 1442-1463.

[10] J. Yan, A. Zhang, L.-Y. Shao, J.-F. Ding, S. He, Simultaneous measurement of refractive index and temperature by using dual long-period gratings with an etching process, IEEE Sens. J. 7 (2007) 1360-1361.

[11] K. Schroeder, W. Ecke, R. Mueller, R. Willsch, A. Andreev, A fibre Bragg grating refractometer, Meas. Sci. Technol. 12 (2001) 757-764.

[12] C. Jesus, P. Caldas, O. Frazao, J.L. Santos, P.A.S. Jorge, J.M. Baptista, Simultaneous measurement of refractive index and temperature using a hybrid fiber Bragg grating/long-period fiber grating configuration, Fiber Integr. Opt. 28 (2009) 440-449.

[13] C. Zhong, C. Shen, Y. You, J. Chu, X. Zou, X. Dong, et al., A polarizationmaintaining fiber loop mirror based sensor for liquid refractive index absolute measurement, Sens. Actuators B 168 (2012) 360-364.

[14] C. Gouveia, G. Chesini, C.M.B. Cordeiro, J.M. Baptista, P.A.S. Jorge, Simultaneous measurement of refractive index and temperature using multimode interference inside a high birefringence fiber loop mirror, Sens. Actuators B 177 (2013) $717-723$.

[15] P. Henderson, Y. Rao, D. Jackson, L. Zhang, I. Bennion, Simultaneous multi-parameter monitoring using a serial fibre-Fabry-Perot array with lowcoherence and wavelength-domain detection, Meas. Sci. Technol. 9 (1998) 1837-1839.

[16] P. Caldas, P.A.S. Jorge, F.M. Araújo, L.A. Ferreira, G. Rego, J.L. Santos, Geometrical effects on the refractive index sensitivity of Mach-Zehnder fibre modal interferometers based on long-period gratings, Meas. Sci. Technol. 20 (2009) 075201.

[17] L.A. Ferreira, A.B.L. Ribeiro, J.L. Santos, F. Farahi, Simultaneous displacement and temperature sensing using a white light interrogated low finesse cavity in line with a fiber Bragg grating, Smart Mater. Struct. 7 (1998) 189-198.

[18] A. Kersey, T. Berkoff, W. Morey, High-resolution fibre-grating based strain sensor with interferometric wavelength-shift detection, Electron. Lett. 28 (1992) $236-238$.

[19] Y.J. Rao, D.A. Jackson, Recent progress in fibre optic low-coherence interferometry, Meas. Sci. Technol. 7 (1996) 981-999.

[20] A.P. Zhang, Z.-G. Guan, S. He, Optical low-coherence reflectometry based on long-period grating Mach-Zehnder interferometers, Appl. Opt. 45 (2006) $1-7$.

[21] M. Jiang, Z.-G. Guan, S. He, Multiplexing scheme for self-interfering longperiod fiber gratings using a low-coherence reflectometry, IEEE Sens. J. 7 (2007) 1663-1667.

[22] A.D. Kersey, T.A. Berkoff, Fiberoptic Bragg-grating differential-temperature sensor, IEEE Photon. Technol. Lett. 4 (1992) 1183-1185.

[23] L.C. Goncalves, G. Gonzalez-Aguilar, O. Frazao, J.M. Baptista, P.A.S. Jorge, Chemical sensing by differential thermal analysis with a digitally controlled fiber optic interferometer, Rev. Sci. Instrum. 84 (2013) 015002.

[24] Y.L. Lo, H.Y. Lai, W.C. Wang, Developing stable optical fiber refractometers using PMDI with two-parallel Fabry-Perots, Sens. Actuators B 62 (2000) 49-54.

[25] Y.L. Lo, C.H. Chuang, Differential optical fiber refractometer based on a pathmatching differential interferometer with temperature compensation, Appl. Opt. 40 (2001) 3518-3524.

[26] S. Murtry, J. Wright, D. Jackson, Sensing applications of a low-coherence fibreoptic interferometer measuring the refractive index of air, Sens. Actuators B 72 (2001) 69-74.

[27] J.H. Osório, L. Mosquera, C.J. Gouveia, C.R. Biazoli, J.G. Hayashi, P.A.S. Jorge, et al., High sensitivity LPG Mach-Zehnder sensor for real-time fuel conformity analysis, Meas. Sci. Technol. 24 (2013) 015102.
[28] G. Salceda-Delgado, D. Monzon-Hernandez, A. Martinez-Rios, G.A. CardenasSevilla, J. Villatoro, Optical microfiber mode interferometer for temperatureindependent refractometric sensing, Opt. Lett. 37 (2012) 1974-1976.

[29] M.I. Zibaii, H. Latifi, M. Karami, M. Gholami, S.M. Hosseini, M.H. Ghezelayagh, Non-adiabatic tapered optical fiber sensor for measuring the interaction between alpha-amino acids in aqueous carbohydrate solution, Meas. Sci. Technol. 21 (2010).

[30] M.I. Zibaii, O. Frazao, H. Latifi, P.A.S. Jorge, Controlling the sensitivity of refractive index measurement using a tapered fiber loop mirror, IEEE Photon. Technol. Lett. 23 (2011) 1219-1221.

[31] H. El-Kashef, The necessary requirements imposed on polar dielectric laser dye solvents, Physica B 279 (2000) 295-301.

[32] E.T. Fogg, A.N. Hixson, A.R. Thompson, Densities, Refractive indexes for ethylene glycol-water solutions, Anal. Chem. 27 (1955) 1609-1611.

[33] P.E. Ciddor, Refractive index of air: new equations for the visible and near infrared, Appl. Opt. 35 (1996) 1566-1573.

\section{Biographies}

Carlos J. Gouveia graduated with a Degree in electronics and telecommunication engineering, in 2007, and the M.Sc. degree in telecommunications and networks, in 2008, from the University of Madeira, Portugal. Currently, he is a PhD student in University of Madeira, work in the Optoelectronics Group of INESC Porto. His research interests are on optical fiber sensors and interrogation systems for chemical and biochemical applications.

Mohammad Zibaii received the M.Sc. and PhD degree in photonics from Laser and Plasma Research Institute, Shahid Beheshti University, Tehran, Iran in 2011. His PhD degree was focused on research in fiber-optic biosensors. He is currently an Assistant Professor of photonics at Laser and Plasma Research Institute of Shahid Beheshti University. His research interest includes development of fiber-optic sensors and label-free fiber optic biosensors for DNA-Drug interaction, Bacteria, and protein in low concentration.

Hamid Latifi received the B.S. degree from California state university in Harvard, and the M.Sc. and PhD degrees from New-Mexico State University, Las Cruces, in 1989 , all in physics, working on interaction of high-energy laser beam aerosols. He was a Postdoctoral Research at Colorado State University in Fort Collins, working on development of sodium Lidar for mesospheric temperature measurement. He joined the Physics Faculty of Shahid Beheshti University (former: National University), Tehran, Iran, in 1991, where he is currently a Professor of physics at Laser and Plasma Research Institute. His current interest includes diode pumped solid state laser, RF-excited $\mathrm{CO}_{2}$ lasers, optical nondestructive testing, and fiber-optic sensors.

Manuel J.B. Marques received a degree in physics from Porto University in 1983, and a PhD degree in physics from the same university in 1991. His thesis work was on nonlinear coupling to optical waveguides. From 1993 to 2001 he was a member of Centro de Física do Porto, where he worked on fiber lasers and integrated optics. He joined INESC Porto, Portugal, in January 2002, where he has worked on fiber lasers and sensors. He has been an assistant professor in the Physics Department of Porto University since 1991. Dr. Marques is a member of the Portuguese Physics Society.

José M. Baptista graduated with a Degree in electrical and computer engineering (telecommunications and computers) from the University of Porto, Porto, Portugal, in 1991, the M.Sc. degree in physics of laser communications from the University of Essex, Colchester, U.K., in 1993, and the PhD degree in electrical and computer engineering from the University of Porto, in 2002. Currently, he is an Associate Professor and President of the Exact Sciences and Engineering Competence Center at the University of Madeira and a Senior Researcher in the Optoelectronics and Electronics Systems Unit at INESC Porto. He is author of 62 journal publications and of two patents. His research interests are in the area of fiber-optic sensors, fiber-optic communications, and fiber-optic technologies.

Pedro A.S. Jorge graduated in applied physics (optics and lasers) from the University of Minho in 1996. He received his M.Sc. in optoelectronics and lasers from the physics department of the University of Porto in 2000. In 2006, he concluded his PhD program at Porto University in collaboration with the department of physics and optical sciences at the University of Charlotte, North Carolina, USA, with work developed in luminescence based optical fiber. From 1997 he has been involved in several research and technology transfer projects related to optical fiber sensing technology, developing new sensing configurations and interrogation techniques for optical sensors, both as a researcher and more recently as project leader and manager. Pedro Jorge is currently a Senior researcher at INESC-Porto where he leads the Biochemical Sensors team exploring the potential of optical fiber and integrated optics technologies in environmental and medical applications coordinating several projects in the area. He has more than 150 publications in the fields of sensors in national and international conferences and peer reviewed journals, is author of 2 book chapters and also holds one patent. 